\title{
Color image encryption via compressive sensing and chaotic systems
}

\author{
Kaige Zhu* and Jinli Cheng \\ School of Computer and Information Engineering, Henan University, Kaifeng 475004, China
}

Keywords: Encryption, Compressive sensing, Chaotic systems.

\begin{abstract}
In this paper, we design a color image encryption algorithm based on chaotic system and block compressive sensing. Firstly, the sparse representation of the plain-image is obtained by an adaptive learning dictionary. Secondly, the key streams are produced from two excellent lowdimensional chaotic maps, where updating the initial values and parameters rely on the SHA-384 and the input image. Thirdly, three measurement matrices of R, G, B components are constructed from the chaotic sequences, respectively. Finally, a random rows and columns diffusion method is performed on the encrypted image. Experimental results and safety analysis prove that the proposed scheme has excellent performance.
\end{abstract}

\section{Introduction}

Compressive Sensing (CS) theory is one of the methods of digital images encryption, which can achieve compression and encryption simultaneously. In [1], a CS image encryption algorithm was proposed, where the measurement matrix is constructed via logistic map. The chaotic system had a pivotal role in constructing the measurement matrix because their chaotic sequences are random and deterministic signal. Subsequently, Gong et al. [2] put forward an image compression and encryption algorithm based on chaotic system, which had a good ability on resistance the known plaintext attacks. However, the measurement matrix was generated from low-dimensional chaotic systems with the simple structures, which greatly reduce the security and the sensitive of the algorithms. To solve this problem, highdimensional chaotic maps were applied in the image encryption methods [3-5]. The Chen's hyperchaotic system was performed on the 2D CS-based image encryption algorithm in [3]. Chai et al. [4] explored a new magnetic controlled memristive chaotic system to construct the circular measurement matrix and encrypt the image, which can enhance the security. Recently, Xu et al. [5] also applied a hyper-chaotic system to encrypt image, which achieved an acceptable compression effects. Unfortunately, these complex chaotic maps increased the computation complexity, and the constructed measurement matrices are single, which would reduce the security and sensibility of cryptosystem. In the current studies, most of them employed the fixed dictionary such as DWT to represent different images sparsely, and the

\footnotetext{
*Corresponding author: 1520156473@qq.com
} 
attained dictionaries are identical and has an influence on the recovering the image.

Motivated by the above analysis, we propose an image encryption algorithm by means of two simple improved chaotic systems, the adaptive learning dictionary algorithm and a random diffusion of rows and columns process. Compared with the existing schemes, our method has a higher security and a better image reconstruction effect. Numerical experiments have verified the feasibility and validity of the proposed algorithm.

\section{Preliminaries}

\subsection{Compressive sensing}

A signal $x$ with size $N \times N$ is sparse in a $N \times N$ transform domain $\psi$, and a measurement matrix $\phi M \times N(M<N)$ which is uncorrelated with $\psi$ is used to yield the measurement $y$ in linear projection [6] as follows:

$$
y=\varphi x=\varphi \psi s=A s
$$

where $A$ is the sensing matrix, $S$ is the sparse coefficient. $x$ can be recovered by:

$$
\min \|s\|_{0} \text { subject to } y=A s \text {. }
$$

Obviously, the signal reconstruction is an ill-posed inverse problem. To overcome this problem, we adopt the Iterative Reweighted Least Squares algorithm (IRLS) [7], which is based on minimum norm with weight iteration.

\subsection{Chaotic systems}

In this paper, two chaotic maps with excellent performance, i.e., LSS and LASM [8], are used to image encryption, which are separately described as follows:

$$
\begin{gathered}
X_{n+1}=\left(r X_{n}\left(1-X_{n}\right)+(4-r) \sin \left(\pi X_{n}\right) / 4\right) \bmod 1, \\
\left\{\begin{array}{c}
Y_{n+1}=\sin \left(\pi \mu\left(Z_{n}+3\right) Y_{n}\left(1-Y_{n}\right)\right) \\
Z_{n+1}=\sin \left(\pi \mu\left(Y_{n+1}+3\right) Z_{n}\left(1-Z_{n}\right)\right),
\end{array}\right.
\end{gathered}
$$

where $r \in(0,4], \mu \in[0,1], X_{n}, Y_{n}, Z_{n} \in(0,1)$.

\section{The proposed encryption scheme}

\subsection{Generating the sparse dictionaries}

Suppose a color image $I$ with size $M \times N$. The R, G, B components of $I$ are denoted as $i m g R, \operatorname{img} G, \operatorname{img} B$, respectively. We divide three components $i m g R, \operatorname{img} G$ and $i m g B$ into $B \times B$ non-overlapping blocks, and convert the image blocks into the vectors with $B^{2} \times 1$. After image segmentation, different image blocks have some similar properties. So we 
calculate the variance values of all blocks, and the image blocks with large variance are selected as the parameters of the Method of Optimal Directions (MOD) algorithm [9], where the number of chose blocks is $N$. And use the MOD algorithm to get the sparse dictionaries $D_{i}$ with size $B^{2} \times N$, where $i=R, G, B$.

\subsection{Generating the key streams for pixel-level diffusion}

Step1 Employ the SHA-384 and the plain image $I$ to update the initial values $x_{0}, y_{0}, z_{0}$ and the parameters $r_{0}, \mu_{0}$ as follows:

$$
\begin{aligned}
& r=r_{0}-\left(h_{1}-h_{2}\right) / 2^{8}, \\
& \mu=\mu_{0}+\left(h_{3}-h_{4}\right) / 2^{8}, \\
& x_{1}=x_{0}-h_{5} \oplus\left(h_{7}-h_{6}\right) / 2^{8}, \\
& y_{1}=y_{0}+\bmod \left(h_{8}+h_{9} / h_{10}, 1\right), \\
& z_{1}=z_{0}-\bmod \left(h_{11} / h_{12}, 1\right),
\end{aligned}
$$

where $h_{i}$ denotes the hash values, $i=1,2, \cdots 12$. $\oplus$ represents an exclusive OR, and $\bmod (x, y)$ returns the remainder after division.

Step 2 Use $r, \mu, x_{1}, y_{1}$ and $z_{1}$ to iterate LSS and LASM for $l_{0}+m \times N$ times, where $l_{0}=1000, m=\frac{M}{B} \times\lceil r \times B\rceil$, and $r$ is the compression ratio of blocks. In order to alleviate the harmful effect of the transient, we discard the former 1000 values and get three key streams $x, y, z$ with length $m \times N$.

Step 3 To enhance the sensitivity of the system, we recombine the sequences $x, y$ and $z$ by:

$$
\begin{aligned}
& x 1=\left[x_{1}, x_{2}, \cdots, x_{m N / 2}, y_{m N / 2+1}, y_{m N / 2+2}, \cdots, y_{3 m N / 4}, z_{3 m N / 4+1}, z_{3 m N / 4+2}, \cdots, z_{m N}\right], \\
& y 1=\left[y_{m N / 2+1}, y_{m N / 2+2}, \cdots, y_{m N}, x_{3 m N / 4+1}, x_{3 m N / 4+2}, \cdots, x_{m N}, z_{m N / 2+1}, z_{m N / 2+2}, \cdots, z_{3 m N / 4}\right], \\
& z 1=\left[z_{1}, z_{2}, \cdots, z_{m N / 2}, y_{m N / 2+1}, y_{3 m N / 4+1}, y_{3 m N / 4+2}, \cdots, y_{m N}, x_{m N / 2+1}, x_{m N / 2+2}, \cdots, x_{3 m N / 4}\right]
\end{aligned}
$$

Step 4 To get the ideal pseudo-random sequences, the chaotic sequences $x 1, y 1$ and $z 1$ are further processed as follows:

$$
X^{\prime}=10^{\alpha} \times x 1-\text { floor }\left(10^{\alpha} \times x 1\right)
$$




$$
\begin{aligned}
& Y^{\prime}=10^{\beta} \times y 1-\text { floor }\left(10^{\beta} \times y 1\right), \\
& Z^{\prime}=10^{\lambda} \times z 1-\text { floor }\left(10^{\lambda} \times z 1\right),
\end{aligned}
$$

where $\alpha=5, \beta=6, \lambda=7$.

Step 5 Sort the sequences $X^{\prime}, Y^{\prime}$ and $Z^{\prime}$ with length $m \times N$ in ascending order, and get the index sequences Index 1 , Index 2 and Index 3 . Similarly, sort respectively the sequences $X^{\prime}, Y^{\prime}$ and $Z^{\prime}$, and obtain the index sequences IndRm, IndGm, IndBm with length $m$ and IndRn, IndGn and IndBn with length $N$.

Step 6 To get the key streams for modifying the pixel values of the image, we convert the sequences Index1, Index 2 and Index 3 into three matrices IndexR, IndexG and IndexB with size $m \times N$, respectively. We quantize three matrices IndexR, IndexG and IndexB into the range of $[0,255]$, and get three matrices $S K R, S K G$ and $S K B$.

\subsection{Encryption process}

Step 1 Input a color image $I$ with $M \times N$, and employ MOD in Section 3.1 to generate the sparse dictionaries.

Step 2 To construct three measurement matrices of R, G, B components, we sample the chaotic sequences $x 1, y 1$ and $z 1$ with intervals $d$, where $d=3$, and get three measurement matrices $\phi R, \phi G$ and $\phi B$ with size $(\lceil r \times B\rceil \times B) \times B^{2}$.

Step 3 The matrices blockmatrix $x_{i}$ are projected and measured by the measurement matrices in column to get measurements $y_{i}$, where $i=R, G, B$. And transform them into three matrices $y R R, y G G$ and $y B B$ with $m \times N$, where $m=\frac{M}{B} \times\lceil r \times B\rceil$.

Step 4 The key streams Index2, Index 1 and Index 3 are applied to shuffle the positions of $y R R, y G G$ and $y B B$, and obtain three $m \times N$ matrices $y R 1, y G 1$ and $y B 1$.

Step 5 To further strengthen the security of the algorithm, the key streams $S K R, S K G$ and $S K B$ are employed to modify the pixel values of $y R 1, y G 1$ and $y B 1$ as follows:

$$
\begin{aligned}
& y R 2(i,:)=y R 1(\operatorname{IndRm}(i),:) \oplus \operatorname{SKR}(i,:), \\
& y G 2(i,:)=y G 1(\operatorname{Ind} G m(i),:) \oplus S K G(i,:), \\
& y B 2(i,:)=y B 1(\operatorname{IndBm}(i),:) \oplus S K B(i,:), \\
& \mathrm{CR}(:, j)=y R 2(:, \operatorname{IndRn}(j)) \oplus \operatorname{SKR}(:, j), \\
& C G(:, j)=y G 2(:, \operatorname{Ind} G n(j)) \oplus \operatorname{SKG}(:, j),
\end{aligned}
$$




$$
C B(:, j)=y B 2(:, \operatorname{IndBn}(j)) \oplus S K B(:, j),
$$

where $1 \leq i \leq m, 1 \leq j \leq N$, the matrices $C R, C G$ and $C B$ are three components of the final encrypted image.

\section{Simulation results}

We choose the 'Lena' image with size $256 \times 256$ as the test image, which is displayed in Fig. 1(a). The initial values are set as follows: $\gamma_{0}=3.578196573421804, \mu_{0}=0.766936472082413$, $x_{0}=0.273642281087165, y_{0}=0.322934593157826, z_{0}=0.608100359832734, B=8$ and the compression ratio of blocks $r$ is 0.4 . In other words, the total compression ratio is $50 \%$, and the encryption results are shown in Fig. 1(b). One can clearly see that the cipher image is noise-like images and the corresponding decrypted image in Fig. 1(c) is almost identical to the original image (Fig. 1(a)). Therefore, our algorithm is feasible and has high reconstruction accuracy.
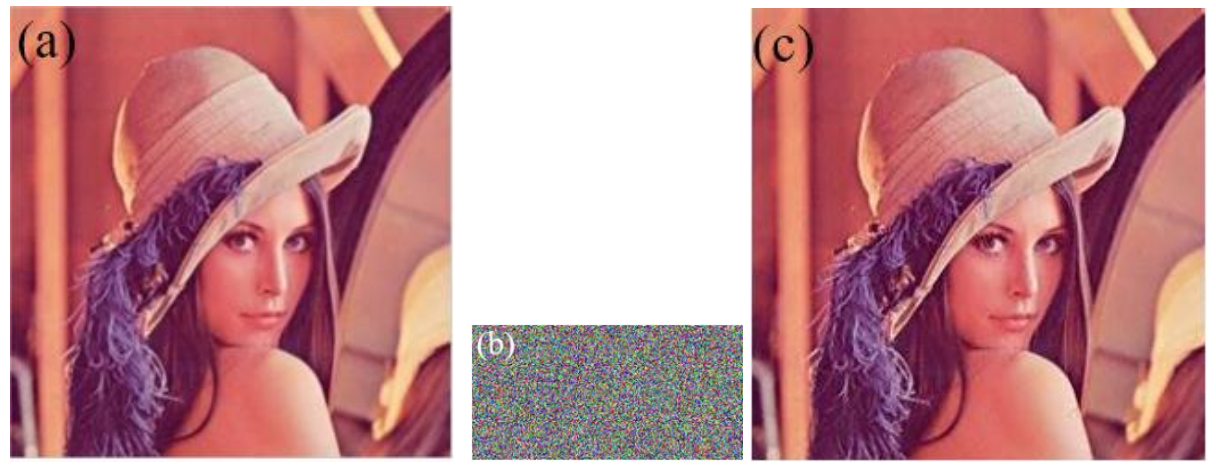

Fig. 1. Experience results: (a) Lena plain-image, (b) The cipher image; (c) The corresponding decrypted image.

\section{Performance and security analysis}

\subsection{Compressive sensing analysis}

The peak signal-to-noise ratio (PSNR) is an important indicator to assess image quality. PSNR is defined as follows:

$$
P S N R=20 \log _{10}\left(\operatorname{MaxI} / \sqrt{\frac{1}{M N} \sum_{i=0}^{M-1} \sum_{j=0}^{N-1}[X(i, j)-Y(i, j)]^{2}}\right),
$$

where MaxI denotes the maximum value, $X$ and $Y$ separately represent the original image and the processed image. Generally, the human eyes cannot differentiate between the original and processed images when $P S N R \geq 30 d B$ [10]. Table 1 provides the PSNR values for the different methods. We can apparently see that our method is superior to other schemes in [4, $5,11,12]$ in terms of reconstructed image quality. 
Table 1. PSNRs (dB) for the different methods.

\begin{tabular}{|c|c|c|c|c|c|c|}
\hline Image & CR & $\operatorname{Ref}[4]$ & $\operatorname{Ref}[5]$ & $\operatorname{Ref}[11]$ & $\operatorname{Ref}[12]$ & Ours \\
\hline \multirow{4}{*}{ Lena } & $25 \%$ & 26.06 & 26.52 & 6.42 & 22.62 & 29.25 \\
\cline { 2 - 7 } & $50 \%$ & 29.82 & 29.23 & 29.23 & 26.87 & 36.38 \\
\cline { 2 - 7 } & $75 \%$ & 29.56 & 29.22 & 33.95 & 30.82 & 43.47 \\
\hline
\end{tabular}

\subsection{Key space analysis}

The designed algorithmm mainly involves the secret keys $\gamma_{0}, \mu_{0}, x_{0}, y_{0}$ and $z_{0}$. If the computational precision is $10^{-15}$, the key space of our method is $10^{75} \approx 2^{250}$, which is larger than those in $[3,5]$. Apparently, the key space of our method is large enough to resist the brute force attacks.

\subsection{Secret key sensitivity analysis}

In the sensitivity analysis, a very slight change $\left(\Delta=10^{-15}\right)$ is applied to the secret key $\gamma_{0}$, for example, $\gamma_{0}^{\prime}=\gamma_{0}-\Delta . \gamma_{0}^{\prime}$ is applied to decrypt the encrypted image Fig. 1(b). Fig. 2 gives the simulation results. From Fig. 2(a), we do not find any information of Lena, and their difference rate between Fig. 1(c) and Fig. 2(a) is more than 99\%. The simulation results show that our algorithm is very sensitive to the secret keys.
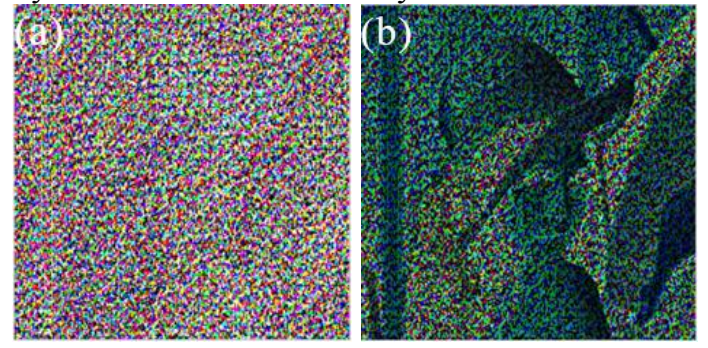

Fig. 2. Key sensitivity analysis: (a) Lena decrypted image with the modified key ${ }^{\prime}$; (b) Differential image between (a) and Fig. 1(c).

\section{Conclusion}

In this paper, an encryption scheme is proposed using compressive sensing and chaotic maps. On the one hand, the scheme uses CS to reduce the size of cipher image and improve the accuracy of image reconstruction. On the other hand, the key streams are utilized to reencrypt the compressed image, which greatly improves the security of encryption algorithm. Experimental results and security analysis have demonstrated that the proposed method has a satisfactory performance.

\section{References}

1. N. Zhou, A. Zhang, F. Zheng, Novel image compression-encryption hybrid algorithm based on key-controlled measurement matrix in compressive sensing, J. Optics \& Laser Technology. 62 (2014) 152-160. 
2. L. Gong, K. Qiu, C. Deng, An image compression and encryption algorithm based on chaotic system and compressive sensing, J. Optics \& Laser Technology. 115 (2019) 257267.

3. J. Chen, Y. Zhang, L. Qi, Exploiting chaos-based compressed sensing and cryptographic algorithm for image encryption and compression, J. Optics \& Laser Technology. 99 (2018) 238-248.

4. X. Chai, X. Zheng, Z. Gan, An image encryption algorithm based on chaotic system and compressive sensing, J. Signal Processing. 148 (2018) 124-144.

5. Q. Xu, K. Sun, C. Cao, A fast image encryption algorithm based on compressive sensing and hyperchaotic map, J. Optics and Lasers in Engineering. 121 (2019) 203-2.

6. E. Candes, J. Romberg, T. Tao, Robust uncertainty principles: Exact signal reconstruction from highly incomplete frequency information, J. arXiv preprint math/0409186 (2004).

7. R. Wolke, H. Schwetlick, Iteratively reweighted least squares: algorithms, convergence analysis, and numerical comparisons, J. SIAM journal on scientific and statistical computing. 9 (1988) 907-921.

8. X. Wu, K. Wang, X. Wang, Lossless chaotic color image cryptosystem based on DNA encryption and entropy, J. Nonlinear Dynamics. 90 (2017) 855-875.

9. K. Engan, S. O. Aase, J. H. Husoy, Method of optimal directions for frame design[C]//1999 IEEE International Conference on Acoustics, Speech, and Signal Processing, Proceedings, ICASSP99 (Cat. No. 99CH36258), IEEE. 5 (1999) 2443-2446.

10. Q. Huynh-Thu, M. Ghanbari, Scope of validity of PSNR in image/video quality assessment. J. Electronics letters. 44 (2008) 800-801.

11. L. Yaru, W. Jianhua, Image encryption based on compressive sensing and variable parameter chaotic mapping, J. Journal of Optoelectronics Laser, Tianjin. 26 (2015) 605610.

12. N. Zhou, A. Zhang, J. Wu, Novel hybrid image compression-encryption algorithm based on compressive sensing, J. Optik-International Journal for Light and Electron Optics. 125 (2014) 5075-5080. 